\title{
Low Dose Electron Holography: First Steps.
}

\author{
Edgar Voelk1 ${ }^{1}$, Rodney Herring ${ }^{2}$, Benjamin Bammes ${ }^{3}$ and David Hoyle ${ }^{4}$ \\ 1. Hitachi High Technologies America, Inc., Clarksburg, MD, USA. \\ 2. University of Victoria, Victoria, Canada. \\ 3. Direct Electron LP, San Diego, CA, USA. \\ 4. Hitachi High Technologies Canada, Inc., Toronto, Canada.
}

One of the key features of electron holography is its capability for providing access to the phase information of the image wave and ultimately to the object wave. This fact can be indicated easily by the definition of the image intensity that is recorded with standard recording devices like CCD cameras: $I(x, y)=|a(x, y) \exp (\mathrm{i} \varphi(x, y))| 2=a^{2}(x, y)$, with $I(x, y)$ the intensity to be recorded, $a(x, y)$ the amplitude of the image wave and $\varphi(x, y)$ the phase of the image wave. Obviously, the phase information $\varphi(x, y)$ is lost but is recoverable with holography [1].

Acquiring electron holograms always has been a technological challenge. On film, both the nonlinearity and the MTF (modulation transfer function) impacted the quality of the hologram as higher order sidebands are generated and higher frequencies are dampened. Although digital cameras are very linear up to at least $70 \%$ to their saturation, the MTF of almost every camera on the market drops to around $10 \%$ or below at the Nyquist limit and for sampling rates (pixels per interference fringe) $s<10$, most MTFs are already below $50 \%$ at the location of the sideband (the main carrier of the holographic information). As a way out, or more as a compromise, electron holograms are recorded highly oversampled and the images obtained from the holograms are often downsized as they contain a lot of empty information due to the oversampling. Alternatively, holograms can be recorded with a binning factor of 2, 4 or even 8. In that case, the large binning factor increases the MTF at the Nyquist limit and oversampling (and empty information) is avoided. Both solutions are far from ideal, as, to draw a simple picture: a $4 \mathrm{k} \times 4 \mathrm{k}$ camera is effectively reduced to a $1 \mathrm{k}$ by $1 \mathrm{k}$ camera or less as to be useful for electron holography. In addition, the DQE (detection quantum efficiency) of CCD cameras in general is $<<1$, simply because vendors create a compromise between a reasonable MTF and a reasonable DQE. It might be reasonable to say there are no dedicated cameras for electron holography. To compare existing CCD cameras for their use for holography, we followed the approach taken in [2] and simulated electron holograms on an electron-by-electron basis.

With the availability of cameras that are exposed directly to the electron beam, like the DE-12 from Direct Electron [3], the holography community is closer than ever to the ideal camera for electron holography. For testing the usability of the DE-12 for electron holography, it was installed temporarily on the Hitachi HF3300V (or 'STEHM') at the University of Victoria [4]. For the data presented in Fig.1, the brightness was adjusted such that an average of 130 electrons per frame at 150fps (frames per second) was obtained for images of the size of 512x512 pixels. Frames were pre-processed using an electron counting algorithm to record the precise location of each incident primary electron in each frame before summing to form the final hologram. The resulting holograms were then evaluated for average counts, fringe contrast and sampling rate. These parameters were then used to simulate the data via HoloWorks [5] by building the hologram from single electron events for an ideal camera. Then both the experimental and simulated holograms were reconstructed and the phase images evaluated after phase-tilt correction. For $1.19 \mathrm{e}^{-}$per pixel, a sampling rate $s=22.9$ and fringe contrast $\mu=64.4 \%$, the 
standard deviation was computed for the central area of each phase image and found to be $2 \pi / 71$ for the experimental data and $2 \pi / 80$ for the simulated data. Experimental and simulated data agree within $15 \%$. Thus we conclude that the DE-12 is very close to an ideal camera for low-dose holography. The lowdose hologram in Fig. $2 \mathrm{a}$, recorded at an average of $4.19 \mathrm{e}^{-}$per pixel, $\mu=44 \%$ and $\mathrm{s}=3.5$ shows the potential of low-dose electron holography: the reconstructed phase image Fig. $2 \mathrm{~b}$ allows the measurement of the film thickness Fig.2c, basically invisible in Fig.2a at an extremely low dose.

\section{References:}

[1] "Introduction to Electron Holography," eds. E Voelk1, LF Allard and DC Joy (Kluwer Academic / Plenum Publishers 1999).

[2] E. Voelkl, Ultramicroscopy, Volume 110, Issue 3, February 2010, Pages 199-210

[3] DE-12, Camera System from Direct Electron, LP, http://www.directelectron.com

[4] University of Victoria, Canada.

[5] HoloWorks, by HoloWerk LLC, http://www HoloWerk.com
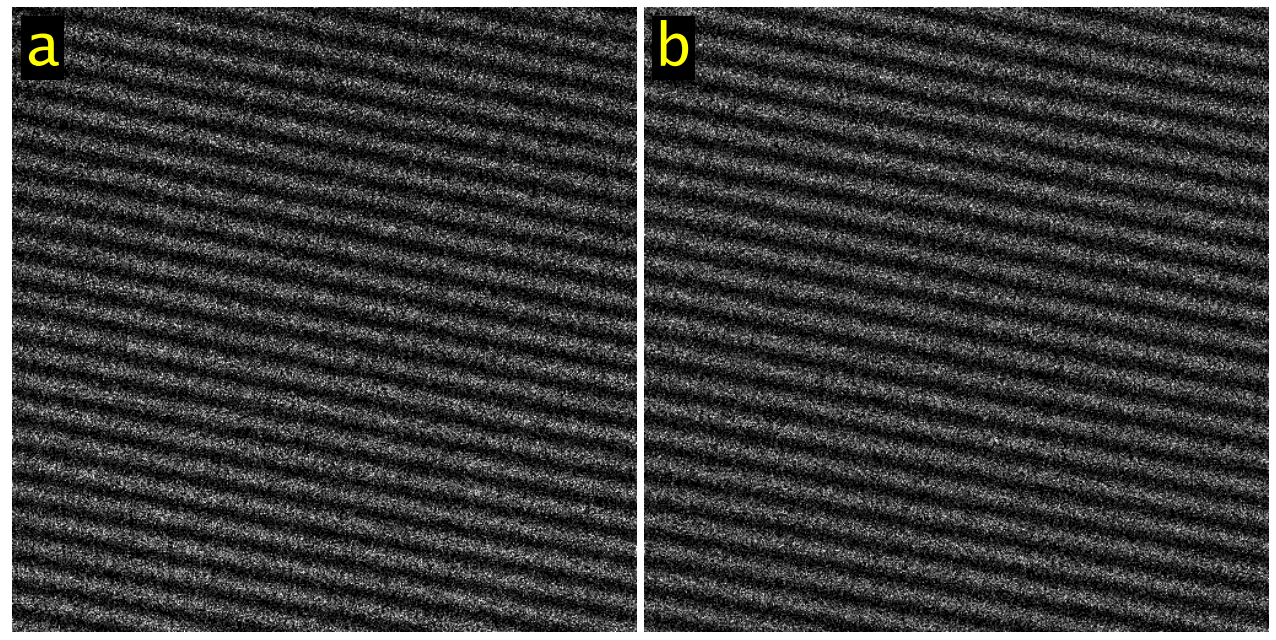

Fig. 1 a: Experimental data composed of 2386 frames recorded at $150 \mathrm{fps}$ and 512 by 512 pixels.

b: Simulated data based on fringe contrast, sampling rate and average pixel value. The standard deviation values within reconstructed phase images agree $<15 \%$.
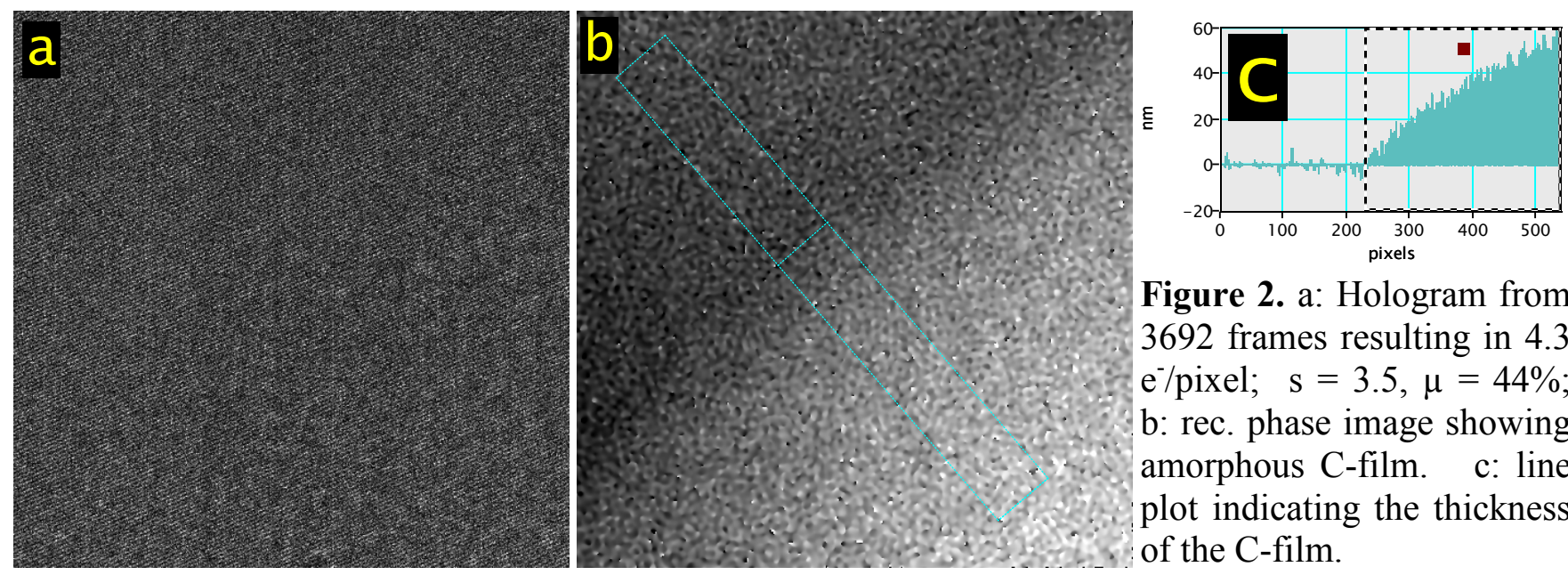

Figure 2. a: Hologram from 3692 frames resulting in 4.3 $\mathrm{e}^{-/ \text {pixel; }} \mathrm{s}=3.5, \mu=44 \%$; b: rec. phase image showing amorphous $\mathrm{C}$-film. c: line plot indicating the thickness of the C-film. 